\title{
SELF-ASSEMBLY OF THERMO AND LIGHT RESPONSIVE AMPHIPHILIC LINEAR DENDRITIC BLOCK COPOLYMERS
}

Hugo García-Juan, ${ }^{\text {a }}$ Aurora Nogales, ${ }^{\mathrm{b}}$ Eva Blasco, ${ }^{\mathrm{c}}$ Juan Carlos Martínez, ${ }^{\mathrm{d}}$ Igor Šics, ${ }^{\mathrm{d}}$ Tiberio A. Ezquerra, ${ }^{\mathrm{b}}$ Milagros Piñol, ${ }^{\mathrm{a}, *}$ Luis Oriol $^{\mathrm{a}, *}$

a Departamento de Química Orgánica, Facultad de Ciencias, Instituto de Ciencia de Materiales de Aragón (ICMA), Universidad de Zaragoza-CSIC, 50009 Zaragoza, Spain.

${ }^{\mathrm{b}}$ Instituto de Estructura de la Materia, IEM-CSIC, C/Serrano 121, 28006 Madrid, Spain

${ }^{\mathrm{c}}$ Present address: Preparative Macromolecular Chemistry, Institut für Technische

Chemie und Polymerchemie, Karlsruhe Institute of Technology (KIT), Engesserstr. 18, 76128 Karlsruhe (Germany)

${ }^{\mathrm{d}}$ Cells-Alba, Carretera, BP 1413, 08290 Cerdanyola del Vallès, Barcelona, Spain

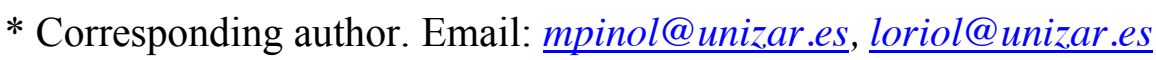




\begin{abstract}
The synthesis and structural characterization of a new dual responsive linear-dendritic block copolymer (LDBC) is presented. The LDBC is constituted by a thermoresponsive linear block from polymethacrylate of oligo- and diethylene glycol, and a light responsive dendron block of bis-MPA decorated at the periphery with 4isobutyloxyazobenzene and alkyl chains in a 50:50 molar ratio. Blocks are coupled together by copper(I) catalyzed alkyne-azide cycloaddition (CuAAC). The ability of the LDBC to form vesicle self-assemblies in water is described, as well as the effect of light and temperature on the vesicles morphology, on the basis of transmission electron microscopy (TEM), dynamic light scattering (DLS) and UV-vis spectroscopy studies. The effect of UV light and temperature on the vesicles structure by SAXS and WAXS conducted on real time is also presented. Finally, the potential use of the vesicles to load and stimuli controlled release of small fluorescent molecules is probed.
\end{abstract}

\title{
KEYWORDS
}

Block copolymer, linear-dendritic, self-assembly, polymeric vesicles, synchrotron 


\section{INTRODUCTION}

Amphiphilic block copolymers (BCs), where one block is hydrophilic and the other hydrophobic, possess the ability to form self-assembled structures in water whose morphology and size depends on the hydrophobic/hydrophilic balance, the length of the blocks, their chemical structure and, in general terms, of their overall architecture. From the different possibilities, polymeric vesicles or polymersomes are of interest as nanocarriers for drug delivery because they are constituted by a particularly robust hydrophobic membrane that can be used to locate hydrophobic guests and an internal hydrophilic cavity that can accommodate hydrophilic ones. ${ }^{[1][2]}$ If stimuli responsive moieties are introduced in such BCs (for example, units sensitive to light, $\mathrm{pH}$ or temperature among the most common), morphology changes can be induced on demand in the vesicles to control the release of the encapsulated compounds. ${ }^{[3]}$ Therefore, the use of stimuli responsive polymersomes offers advantages such as the reduction of the amount of drug needed, a better dissolution of poorly soluble drugs and its controlled release.

From different stimuli, light is a very appealing one because it can be accurately operated in time and space. ${ }^{[4]}$ The light response of certain materials is associated to the presence of light active groups such as azobenzene that isomerizes under illumination. Temperature is also a very interesting stimulus, in particular for bioapplications, since unhealthy tissues might show slightly higher temperature than healthy ones. There are polymers, which are soluble in water, that become insoluble when heated over a certain temperature, called lower critical solution temperature (LCST). Among them, poly[oligo(ethylene glycol) methacrylate]s present good thermo-responsive properties and biocompatibility, making them ideal for biomedical applications. ${ }^{[5]}$ Lutz et al. have described a family of copolymers from oligo- and diethylene glycol methacrylates 
(OEGMA and DEGMA, respectively), which have a tunable LCST depending on the molar ratio between monomers, being this value close to physiological temperature when DEGMA/OEGMA molar ratio is around $85: 15 .^{[6]}$

In the last few years stimuli responsive linear-dendritic block copolymers (LDBCs) have been explored where the well-defined periphery of the dendritic block makes them perfect systems to perfectly control the incorporation of functionalization. ${ }^{[7]}$ From the several possibilities, we have focused our attention on the probably most versatile synthetic strategy for LDBCs which is the coupling of the two constitutive blocks using the copper(I) catalyzed alkyne-azide cycloaddition (CuAAC), where each block is synthetized separately having either azide or alkyne functional groups. Linear polymer chains with terminal alkyne groups are easily approachable by atom transfer radical polymerization (ATRP) using an appropriate initiator. ${ }^{[8]}$ The synthesis of a dendron with an azide group at the focal point and light sensitive moieties is also affordable even if it is more demanding in relation to the number of synthetic and purification steps. Accordingly, amphiphilic LDBCs have been reported from 2,2di(hydroxymethyl)propionic acid (bis-MPA) dendrons with peripheral 4-cyano or 4alkoxyazobenzene units and poly(ethylene glycol) (PEG) as linear block. These LDBCs formed vesicles in water when the $4^{\text {th }}$ bis-MPA dendron generation was coupled

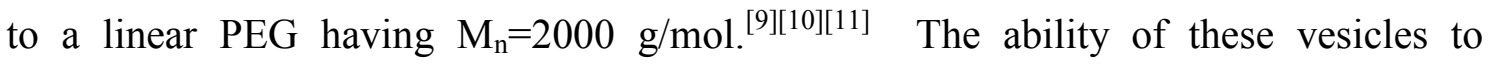
encapsulate and release under UV light trigger of both hydrophobic and hydrophilic fluorescent probes was confirmed in particular when 4-isobutyloxyazobenzene was used as chromophore as facilitates the response to light compared to 4-cyanoazobenzene under lower intensity UV illumination. Furthermore, our results showed that cofunctionalization of the dendron with 4-isobutyloxyazobenzene and long alkyl chains 
facilitates the azobenzene photoisomerization and, therefore the response to light, because chromophore aggregation is restricted. ${ }^{[1]}$

This work reports on the synthesis and characterization of light and thermoresponsive LDBCs combining ATRP and CuAAC chemistries (Figure 1). The $4^{\text {th }}$ generation of a bis-MPA based dendron functionalized at the periphery with 4-isobutyloxyazobenzene and stearic acid in a 50:50 molar ratio will be used as it has been demonstrated optimal performance upon illumination. For the linear block, we intend to prepare a thermoresponsive copolymer of DEGMA and OEGMA having a molar mass close to $2000 \mathrm{~g} / \mathrm{mol}$ as in the linear PEG of previous works, and a LCST close to physiological temperature.

X-ray scattering techniques provide information about the structure and molecular conformations at different length scales. Wide Angle X-Ray Scattering (WAXS) offers the possibility to obtain information about molecular ordering and conformations on the scale of tenths of nanometers. Small Angle X-Ray Scattering (SAXS), on the other hand, allows one to analyze the structure developed over the length scale of tens of nanometers and it is of particular value when studying supramolecular organization. ${ }^{[12]}$ Synchrotron radiation offers the possibility to follow by SAXS and WAXS real time changes in both the conformation and the structure. ${ }^{[13]}$ Therefore, we will explore the ability of the polymers to self-assemble into vesicles and their response to external stimuli as light and temperature with the aid of X-ray scattering techniques.

\section{EXPERIMENTAL SECTION}

\subsection{Materials}

The $4^{\text {th }}$ generation of a bis-MPA based dendron containing 4-isobutyloxy-4'oxyazobenzene units and octadecyl chains randomly distributed at the periphery in a 
50:50 molar ratio, $\mathrm{d}(\mathrm{iso} \mathrm{AZO} / \mathrm{C} 18)$, was synthesized as previously reported. ${ }^{[11]}$ Oligo(ethylene glycol) methyl ether methacrylate (OEGMA) $\left(M_{\mathrm{n}}=475 \mathrm{~g} / \mathrm{mol}\right.$ and $8-9$ ethylene oxide units) and di(ethylene glycol) methyl ether methacrylate (DEGMA) were purchased from Sigma-Aldrich and passed through a column of basic alumina to remove the radical inhibitor. The ATRP initiator 3-(trimethylsilyl)prop-2-ynyl 2bromo-2-methylpropanoate was prepared according to the procedure described in the literature. ${ }^{[14]}$ Ethanol was dried with $\mathrm{KOH}$ and distilled before used. $N, N$ dimethylformamide (DMF) was dried over $4 \AA$ molecular sieves and distilled before used. All other reagents were purchased in Sigma-Aldrich and used as received.

\subsection{Characterization techniques}

${ }^{1} \mathrm{H}$ NMR spectra were recorded on a Bruker AV-400 spectrometer at $400 \mathrm{MHz}$. IR spectra were obtained on a Bruker Tensor 27 or a Nicolet Avatar 360-FT-IR spectrometer, using $\mathrm{KBr}$ pellets for solid compounds and $\mathrm{NaCl}$ for oils. Size exclusion chromatography (SEC) was performed on a Waters e2695 Alliance liquid chromatography system equipped with a Waters 2424 evaporation light scattering detector using two Ultrastyragel ${ }^{\circledR}$ columns (HR4 and HR2) of 500 and $10^{4} \AA$ pore size from Waters, THF at a flow rate of $1 \mathrm{~mL} / \mathrm{min}$ and calibrated against poly(methyl methacrylate) (PMMA) standards. Fluorescence spectra were registered using a Perkin Elmer LS 50B spectrometer. UV-Vis spectra were recorded on an ATI-Unicam UV4200 spectrophotometer. Thermogravimetric analyses (TGA) were performed on a Q5000IR from TA instruments under nitrogen at a $10^{\circ} \mathrm{C} / \mathrm{min}$ heating rate using $5-10$ mg of the sample. Differential Scanning Calorimetry (DSC) was performed with a DSC Q2000 from TA Instruments with powdered samples (2-5 mg). Transmission electron microscopy (TEM) studies were carried out in a JEOL-2000 FXIII electron microscope operating at $200 \mathrm{kV}$. DLS measurements were carried out in a Malvern Instrument 
Nano ZS using a He-Ne laser with a $633 \mathrm{~nm}$ wavelength and a detector angle of $173^{\circ}$ at $25^{\circ} \mathrm{C}$.

\subsection{Synthesis of the thermoresponsive linear block}

Details are given for the block (OEG-stat-DEG)_3500 as an example: OEGMA $(3.15 \mathrm{~g}$, $6.6 \mathrm{mmol})$, DEGMA (5.0 g, $26.6 \mathrm{mmol}), \mathrm{CuBr}(478 \mathrm{mg}, 3.3 \mathrm{mmol}), 2$,2'-bypyridine (bipy) (1.037 g, $6.6 \mathrm{mmol})$ and 3-(trimethylsilyl)prop-2-ynyl 2-bromo-2methypropanoate $(920 \mathrm{mg}, 3.3 \mathrm{mmol})$ were dissolved in deoxygenated ethanol $(9 \mathrm{~mL})$ into a Schlenk flask. The flask was degassed by three freeze-pump-thaw cycles and flushed with argon. The polymerization was conducted at $60^{\circ} \mathrm{C}$ for $10 \mathrm{~min}$. Then, flask was opened to the air and cooled into liquid nitrogen to quench the reaction. The reaction crude was diluted with $\mathrm{THF}$ and passed through a column of neutral alumina to remove the catalyst. THF was evaporated and the residue dialyzed against water with a SpectraPor3 membrane $(\mathrm{MWCO}=1000 \mathrm{~g} / \mathrm{mol})$ for 5 days at room temperature. Finally water was eliminated by lyophilization to yield the polymer as colorless oil. IR $(\mathrm{NaCl}$, $\left.\mathrm{cm}^{-1}\right): 2877\left(\mathrm{Csp}^{3}-\mathrm{H}\right), 1732(\mathrm{C}=\mathrm{O}), 1246(\mathrm{C}-\mathrm{O}) .{ }^{1} \mathrm{H}$ NMR $\left(400 \mathrm{MHz}, \mathrm{CDCl}_{3}\right) \delta(\mathrm{ppm})$ : 4.63-4.52 (m, 2H, $\left.-\mathrm{COO}-\mathrm{CH}_{2}-\mathrm{C} \equiv \mathrm{C}-\mathrm{Si}\left(\mathrm{CH}_{3}\right)_{3}\right), 4.06\left(\mathrm{~s}, \mathrm{COO}-\mathrm{CH}_{2}-\right), 3.60\left(\mathrm{~m},-\mathrm{CH}_{2}-\mathrm{O}\right)$, $3.52\left(\mathrm{~m},-\mathrm{CH}_{2}-\mathrm{O}\right), 3.35\left(\mathrm{~s}, \mathrm{O}-\mathrm{CH}_{3}\right), 1.86\left(\mathrm{~s},-\mathrm{CH}_{2^{-}}\right), 1.77\left(\mathrm{~s},-\mathrm{CH}_{2^{-}}\right), 1.10\left(\mathrm{~s},-\mathrm{CH}_{3}\right), 1.00$ (s, $\left.-\mathrm{CH}_{3}\right), 0.84\left(\mathrm{~s},-\mathrm{CH}_{3}\right), 0.12\left(\mathrm{~s}, 9 \mathrm{H},-\mathrm{Si}\left(\mathrm{CH}_{3}\right)_{3}\right)$

\subsection{Synthesis of the LDBC (OEG-stat-DEG)_3500-block-d(isoAZO/C18)}

Previous to the coupling reaction, the trimethylsilyl protective group of the terminal alkyne of (OEG-stat-DEG)_3500 was removed using the following procedure: (OEGstat-DEG)_3500 (500 mg, $0.156 \mathrm{mmol})$ and acetic acid (140 mg, $0.234 \mathrm{mmol})$ were dissolved in dry THF ( $2 \mathrm{~mL})$ under argon atmosphere. The flask was immersed into an ice bath and then, tetrabutylammonium fluoride (TBAF) $(407 \mathrm{mg}, 1.560 \mathrm{mmol})$ in dry THF ( $1 \mathrm{~mL})$ was dropwise added. The reaction was maintained for $48 \mathrm{~h}$. The reaction 
crude was diluted with THF and passed through a neutral alumina column to remove TBAF excess. THF was evaporated to render the alkyne terminated polymer coded as (OEG-stat-DEG)_3500-C $\equiv \mathrm{CH}$ as a colorless oil. Yield: 95\%. IR $\left(\mathrm{NaCl}, \mathrm{cm}^{-1}\right): 2877$ $\left(\mathrm{Csp}^{3}-\mathrm{H}\right), 1732(\mathrm{C}=\mathrm{O}), 1246(\mathrm{C}-\mathrm{O}) .{ }^{1} \mathrm{H}$ NMR (400 MHz, $\left.\mathrm{CDCl}_{3}\right) \delta(\mathrm{ppm}):$ 4.65-4.55 (m, $\left.2 \mathrm{H},-\mathrm{COO}-\mathrm{CH}_{2}-\mathrm{C} \equiv \mathrm{CH}\right), 4.06\left(\mathrm{~s}, \mathrm{COO}_{-} \mathrm{CH}_{2}-\right), 3.60\left(\mathrm{~m},-\mathrm{CH}_{2}-\mathrm{O}\right), 3.52\left(\mathrm{~m},-\mathrm{CH}_{2}-\mathrm{O}\right)$, $3.35\left(\mathrm{~s}, \mathrm{O}-\mathrm{CH}_{3}-\mathrm{O}\right), 1.86\left(\mathrm{~s},-\mathrm{CH}_{2}-\right), 1.77\left(\mathrm{~s},-\mathrm{CH}_{2}-\right), 1.10\left(\mathrm{~s},-\mathrm{CH}_{3}\right), 1.00\left(\mathrm{~s},-\mathrm{CH}_{3}\right), 0.84$ $\left(\mathrm{s},-\mathrm{CH}_{3}\right)$. For the $\mathrm{CuAAC}$ coupling reaction, (OEG-stat-DEG)_3500-C $\equiv \mathrm{CH}(137 \mathrm{mg}$, $0.042 \mathrm{mmol}), \mathrm{d}($ isoAZO/C18) (150 mg, $0.021 \mathrm{mmol}), \mathrm{CuBr}(6.05 \mathrm{mg}, 0.042 \mathrm{mmol})$ and $N, N, N$ ', $N$ ', $N$ ' '-pentamethyldiethylene triamine (PMDETA) (7.3 mg, $0.042 \mathrm{mmol}$ ) were dissolved in DMF (2 mL) into a Schlenk tube. The reaction mixture was degassed by three freeze-pump-thaw cycles and flushed with argon. The reaction was carried out for $72 \mathrm{~h}$ at $40^{\circ} \mathrm{C}$. Then, reaction mixture was diluted with dichloromethane and filtered through a column of neutral alumina to eliminate copper. Dichloromethane was evaporated and the residual DMF solution precipitated into cold ethanol. Yield: $82 \%$. IR $\left(\mathrm{NaCl}, \mathrm{cm}^{-1}\right) 2921\left(\mathrm{Csp}^{3}-\mathrm{H}\right), 1734(\mathrm{C}=\mathrm{O}), 1599$ (C-CAr), 1471 (C-CAr), 1245 (CO). ${ }^{1} \mathrm{H}$ NMR (400 MHz, $\left.\mathrm{CDCl}_{3}\right) \delta(\mathrm{ppm}) 7.83,6.95,5.14,4.24,4.20,4.10,3.97,3.76$, $3.64,3.55,3.39,2.33,2.27,2.10,1.79,1.66,1.56,1.48,1.25,1.04,0.86$

\subsection{LCST determination}

LCST of the thermoresponsive block were measured by DSC during the heating of a $10 \% \mathrm{w} / \mathrm{w}$ aqueous polymer solution at $10{ }^{\circ} \mathrm{C} / \mathrm{min}$ from $10{ }^{\circ} \mathrm{C}$ to $80{ }^{\circ} \mathrm{C}{ }^{[15]}$ and determined from temperature of the peak maximum in the first derivate of the heat flow curve and corroborated by visual inspection when heating the polymer solution in a thermostatized bath.

\subsection{Formation and characterization of self-assemblies}


Self-assemblies were prepared by the co-solvent method using THF/water. (OEG-statDEG)_3500-block-d(isoAZO/C18) (5 mg) was first dissolved in THF (1 mL) and $10 \mu \mathrm{L}$ portions of Milli-Q water were gradually added, while measuring the loss of intensity of transmitted light (turbidity) at $650 \mathrm{~nm}$ due to aggregation as a function of the water content. When turbidity suddenly increased and once a constant value was reached, the suspension was dialyzed against water for 4 days.

Morphology of the self-assemblies was determined by transmission electron microscopy (TEM) by casting $10 \mu \mathrm{L}$ of a $1 \mathrm{mg} / \mathrm{mL}$ assemblies suspension over a TEM grid. Water was removed by capillarity using a filter paper, the sample was stained with uranyl acetate and the grid was dried under vacuum overnight.

Thermodynamic stability of the self-assemblies was evaluated determining the critical aggregation concentration (CAC) by monitoring fluorescence of Nile Red as probe. Accordingly, $120 \mu \mathrm{L}$ of a $5 \times 10^{-6} \mathrm{M}$ Nile Red solution in dichloromethane were added to a series of vials, and then the solvent evaporated. Afterwards, to each vial, $600 \mu \mathrm{L}$ of a self-assemblies suspension of concentration ranging from 1 to $10^{-4} \mathrm{mg} / \mathrm{mL}$ were added so the final concentration of Nile Red was $10^{-6} \mathrm{M}$. The suspensions were stirred overnight to reach equilibrium, and then fluorescence was measured. Emission spectra were registered from 560 to $700 \mathrm{~nm}$ by exciting at $550 \mathrm{~nm}$. The emission intensity at $606 \mathrm{~nm}$ was represented against logarithm of the LDBC concentration showing a nonlinear dependence. The CAC was determined at the intersection point of the lower horizontal and the slope tangent.

The particle size and distribution of the assemblies were determined by dynamic light scattering (DLS). The self-assemblies concentration was close to $0.05 \mathrm{mg} / \mathrm{mL}$ and size measurements were performed at least three times on each sample to ensure consistency. 


\subsection{Irradiation studies}

Assemblies suspensions were irradiated for different time periods under ambient conditions using a Phillips PL-S 9W lamp placed at a distance of $10 \mathrm{~cm}$. After irradiation, samples were kept at room temperature in the dark.

\subsection{Loading of Rhodamine $B$ into the vesicles}

LDBCs were dissolved in THF and a solution of Rhodamine B in water was added gradually to induce the self-assembly. Concentration of Rhodamine B must be adjusted so that the end the dye concentration was 5:1 respect the LDBC. The final solution was dialyzed against water in order to remove THF and non-encapsulated Rhodamine B.

\subsection{Confocal microscopy studies}

$5 \mu \mathrm{L}$ of vesicles suspension was deposited onto a glass slide and a coverslip was placed over the sample. The sample was sealed to avoid water evaporation. Samples were observed with an Olympus FV10i confocal microscope. Measurements of the fluorescent spots were performed with the ImageJ software.

\subsection{Small and wide angle X-ray scattering experiments (SAXS and WAXS)}

Experiments were performed at beamline BL11-NCD (ALBA, Spain) using a wavelength of $0.1 \mathrm{~nm}$. The SAXS detector (ADSC, Quantum 210r CCD, pixel size 102 $\mu \mathrm{m})$ was located at $6.430 \mathrm{~m}$ distance from the sample position. For the WAXS measurements a LX255-HS Rayonix detector (40x40 $\mu \mathrm{m}$ pixel size) was used. The exposure time was $2 \mathrm{~s}$. For experiments performed upon heating and cooling ramps, a capillary containing the sample was inserted into a Linkam THMS600 hot stage. Simultaneous WAXS and SAXS patterns were acquired while the sample was heated from $20^{\circ} \mathrm{C}$ to $60^{\circ} \mathrm{C}$ at a controlled rate of $1{ }^{\circ} \mathrm{C} / \mathrm{min}$. Experiments under UV irradiation were performed at room temperature in the following way. WAXS and SAXS patterns were collected while the sample was irradiated with a UV lamp (Phillips PL-S 9W) 
placed at a distance of $10 \mathrm{~cm}$. The angular (q-axis) calibration was obtained by measuring standard samples of Silver Behenate for SAXS and $\mathrm{Cr}_{2} \mathrm{O}_{3}$ for WAXS respectively. In these experiments the scattering from water and that of the containing capillary was subtracted from the total scattering contribution.

\section{RESULTS AND DISCUSSION}

\subsection{Synthesis and characterization of thermoresponsive linear polymers}

Thermoresponsive copolymers, (OEG-stat-DEG), were synthetized by ATRP using $\mathrm{CuBr} /$ bipy in ethanol at $60^{\circ} \mathrm{C}$, as described by Lutz, but using an initiator having an alkyne-protected group. ${ }^{[6]}$ Several polymerization experiments were carried out in order to adjust the composition of the linear block to the desired value (see supporting information Table S1). $M_{\mathrm{n}}$ and OEGMA/DEGMA molar proportion were determined by ${ }^{1} \mathrm{H}$ RMN by comparing relative integration of the peak at $0.12 \mathrm{ppm}$ corresponding to $-\mathrm{Si}\left(\mathrm{CH}_{3}\right)_{3}$ end chain radicals (labelled as ' $a$ ' in Figure 2) and that of those at $3.35 \mathrm{ppm}$ (labelled as ' $b$ ' in Figure 2) and 3.45-3.70 ppm, (labelled as ' $c$ ' in Figure 2) corresponding to methyloxy $-\mathrm{OCH}_{3}$ protons and methylenoxy $-\mathrm{CH}_{2} \mathrm{O}-$ protons of the repeating units. In this set of experiments, it was possible to adjust $M_{\mathrm{n}}$ to 3500 after 10 min limiting the monomer/initiator ratio, obtaining a 76:24 DEG/OEG repeating units proportion. The LCST of this (OEG-stat-DEG)_3500 is $42^{\circ} \mathrm{C}$ as was detected by DSC and in accordance with previous results. ${ }^{[5]}$

\subsection{Synthesis and characterization of the LDBC (OEG-stat-DEG)_3500-block- d(isoAZO/C18)}

According to the initial approach of work, the block (OEG-stat-DEG)_3500 was chosen for next step in the synthesis of the aimed LDBC. First, the terminal alkyne was 
deprotected using TBAF while effectiveness of the reaction was verified by ${ }^{1} \mathrm{H}$ RMN by following the disappearance of the signal at $0.12 \mathrm{ppm}$. Then, the LDBC (OEG-statDEG)_3500-block-d(isoAZO/C18) was prepared by CuAAC using the $4^{\text {th }}$ generation of a bis-MPA dendron, $\mathrm{d}(\mathrm{isoAZO} / \mathrm{C} 18)$, functionalized at the periphery with 4isobutyloxyazobenzene units and aliphatic chains in a 50:50 molar proportion (Figure 1). A two-fold excess of the linear block was used to facilitate the completeness of the block coupling reaction. This excess was easily removed and recovered by precipitation of the final LDBC into cold ethanol.

The LDBC and its precursors were analyzed by SEC and the curves show a shifting to lower retention time of the $\mathrm{LDBC}$ respect to the starting blocks (see supporting information Figure S1). By ${ }^{1} \mathrm{H}$ RMN spectroscopy (Figure 3c), it was observed the disappearance of the signal at $\delta=3.25 \mathrm{ppm}$ corresponding to $-\mathrm{CH}_{2}-\mathrm{N}_{3}$ protons of the dendron block (Figure 3a, inset), as well as the signal at $\delta=4.62 \mathrm{ppm}$ corresponding to methylenic protons $-\mathrm{COO}-\mathrm{CH}_{2}-\mathrm{C} \equiv \mathrm{CH}$ of the linear block (Figure $3 \mathrm{~b}$, inset). By IR spectroscopy, neither a band corresponding to an azide at $2097 \mathrm{~cm}^{-1}$ nor a band corresponding to an alkyne at 2260-2100 $\mathrm{cm}^{-1}$ was observed (see supporting information Figure S2). On the whole, traces of residual blocks were discarded.

\subsection{Self-assembly properties of (OEG-stat-DEG)_3500-block-d(isoAZO/C18) in water and effect of light and temperature on the self-assemblies morphology}

LDBC were assembled by the co-solvent method using THF-water while measuring turbidity (see supporting information Figure S3). TEM photographs of stained samples showed the formation of vesicles (Figure $4 a$ ) with hydrodynamic diameter $\left(D_{h}\right)$ values of around $70 \mathrm{~nm}$ and a minor population of $300 \mathrm{~nm}$ according to DLS (see supporting information Figures S4). 
The critical aggregation concentration (CAC) in water was determined by fluorescence using Nile Red, which exhibit a high fluorescence when solubilized into hydrophobic environments such as the interior of the vesicle membrane. Accordingly, Nile Red was equilibrated with dispersions of the LDBC of different concentration and the fluorescence spectra registered from 560 to $700 \mathrm{~nm}$ obtaining a CAC of $35 \mu \mathrm{g} / \mathrm{mL}$, which is in concordance with values for similar LDBCs. ${ }^{[16]}$

The UV-vis spectrum of the vesicles suspension in water $(0.5 \mathrm{mg} / \mathrm{mL})$ was recorded (Figure 5) and compared with that recorded for the polymer in chloroform solution. The maximum absorption band was located at $360 \mathrm{~nm}$, which matches the LDBC main band in chloroform solution due to a $\pi-\pi^{*}$ transition. Besides, two shoulders at 344 and $380 \mathrm{~nm}$ were attributed to the presence of $\mathrm{H}$ - and J-aggregates, respectively.

After exposure to UV-light, a significant decrease and blue-shift of main absorption band, accompanied by an increase of the absorbance at $450 \mathrm{~nm}$, was observed in the UV-vis spectra due to $E$-to- $Z$ photoisomerization (Figure 5). Minor changes were observed after 2 min and a stationary state was reached after 5 min of irradiation. After $24 \mathrm{~h}$ in the dark, a partial recovery of original absorption spectra was observed. ${ }^{[11]}$

The effect of the light and the temperature on the vesicles morphology was investigated by TEM. Upon UV irradiation for $10 \mathrm{~min}$, TEM images showed how vesicles got disrupted and large amounts of unstructured were detected (Figure 4b).

To evaluate the effect of the temperature, an aqueous suspension of the vesicles was heated for 1 hour above the LCST of the thermoresponsive block, assuming that LCST for the linear block of LDBC has a similar value than the block before coupling. A sample for TEM inspection was prepared by casting a drop onto a TEM grid without cooling and images showed the destruction of the vesicles (Figure 4c). Additionally, 
vesicles were heated above LCST and slowly cooled to room temperature, but fully recovery of the vesicles was not observed (Figure $4 \mathrm{~d}$ ).

\subsection{Effect of the UV light and temperature on the vesicles structure by $X$ ray scattering techniques}

(a) Modifications in the vesicles structure due to UV illumination. Figure 6 shows the small angle scattering profile as a function of the module of the reciprocal lattice vector $q(q=4 \pi / \lambda \sin (\theta)$, being $2 \theta$ the scattering angle) from the (OEG-statDEG)_3500-block-d(isoAZO/C18) polymeric vesicles, after subtraction of the water and capillary scattering, before and after $10 \mathrm{~min}$ UV illumination time. In the studied $q$ range, no Bragg peaks were observed. The main features of the SAXS profile were the existence of an initial region at low $q$, where the intensity follows a $q^{-2}$ trend, followed by an intermediate $q$ region, where $q^{-4}$ dependency was observed. Finally a bump centered at $q=0.8 \mathrm{~nm}^{-1}$ was also detected.

In a first approach, this type of scattering is compatible with the presence of the vesicle observed by TEM. ${ }^{[17][18]}$ Due to the limited $q$ range of the experiment, the scattering curve could not be fitted properly. However, based on TEM, a globular shape of the scattering objects can be considered and, therefore, SAXS profiles can be analyzed by using the Guinier approach for globular objects. ${ }^{[19]-[22]}$ At lower $q$, there should be a Guinier region, from which the radius of gyration of the particle can be estimated. The scattering for this regime follows the so called Guinier law $I q=G \exp -q^{2} R_{g}^{2} 3$ where $G$ is the Guinier prefactor and $R_{g}$ is the radius of gyration. ${ }^{[12][21][22]}$ The inset in figure 6 shows the Guinier plot from which $R_{g}$ is estimated. By assuming the vesicle shape to be an sphere, the diameter of it can be obtained as $D=2 \cdot(5 / 3)^{1 / 2} R_{g} .^{[19]-[22]}$ In 
our case a value for the diameter of $\approx 79 \mathrm{~nm}$ was obtained at room temperature. This value agrees rather well the estimates obtained by TEM and DLS.

Figure 7 shows the dependency of the vesicle diameter, as estimated from SAXS, with UV illumination time. The size of the vesicles was modified during UV illumination by exhibiting a decrease on increasing the illumination time.

Simultaneous with the previously described SAXS experiments under UV illumination, WAXS profiles, after water subtraction, are presented in figure 8 . The WAXS profile of the vesicles presents a broad and composed maximum, which is typical from amorphous polymers. The main peak (located at $q=19 \mathrm{~nm}^{-1}$ for the initial sample) correspond to interchain structural correlations, that indicates an average correlation distance of $d_{\text {inter }}=2 \pi / q_{\text {inter }}$ of $3.3 \AA$. Higher $q$ peaks can be attributed to intrachain structural correlations. ${ }^{[23]}$ Several authors have used $d_{\text {inter }}$ to study changes occurring around the glass transition temperature. ${ }^{[24][25]}$ In the present case, we observed that, UV illumination induced a shift of the main peak (the interchain correlation peak) towards higher $q$ values (smaller correlation distances). The dependency of the average interchain correlation distances with UV illumination time is shown in Figure 9. The average distance between chains, $d_{\text {inter }}$, decreases with UV irradiation by approximately $6.5 \%$. This fact could be associated to some sort of distortion of the vesicle wall by the action of the UV illumination, and it is parallel to the decrease in the vesicle size observed by SAXS (Figure 7).

(b) Modifications in the structure due to temperature. Increasing temperature had also a significant effect in the WAXS profiles of the polymer assembled in vesicles. Figure 10 shows WAXS patterns at different temperatures on heating and cooling runs. Increasing temperature produced changes in the intermolecular distance between chains. However, as temperature increased $d_{\text {inter }}$ decreased, indicating that the behavior cannot 
simply be explained by thermal expansion. Increasing the temperature seems to produce a densification of the polymer chains probably due to the hydrophilic-tohydrophobic transition at the LCST of the thermoresponsive block and the consequent collapse of the vesicles. Upon cooling, the initial $d_{\text {inter }}$ was recovered. Within the experimental limit, this change was monotonic, and no special transition temperature was observed (see Figure 11a).

However, the intensity of the peak followed a more complex variation with temperature (see Figure 11b). As temperature increased, the intensity initially increased slightly up to $29^{\circ} \mathrm{C}$, to suddenly decrease, in the temperature range between $30-40{ }^{\circ} \mathrm{C}$ and finally kept on decreasing slightly. This trend in intensity was nearly reversible, observing the same step like behavior in the temperature range between $30-40{ }^{\circ} \mathrm{C}$ upon cooling. The fact that trend was not completely reversible might be related with TEM observations where fully recovery of the vesicles upon cooling below LCST was not observed.

\subsection{Encapsulation and thermo- and photoinduced release of molecular probes}

As it was mentioned, vesicles are able to encapsulate Nile Red, a hydrophobic fluorescent probe, in the interior of the membrane. A suspension of vesicles loaded with Nile Red was irradiated with UV light and the fluorescence spectra recorded at different exposure times. The initial emission markedly decreased upon irradiation during the first $5 \mathrm{~min}$ (Figure 12a). Decreasing of the emission is indeed related to the increase in polarity of the medium where Nile Red is located, which can be either due to the diffusion of the Red Nile to aqueous medium or to the great increase of polarity of the membrane associated with the $E$-to- $Z$ photoisomerization. Because after storing the irradiated sample at room temperature for $24 \mathrm{~h}$ in the dark the initial emission intensity 
is only partially recovered, it can be assumed that Nile Red is at least partially released by light stimulation.

A suspension of vesicles loaded with Nile Red was also heated above LCST for $30 \mathrm{~min}$. However in this case, the emission spectra recorded before and after heating (Figure 12b) are identical, despite the morphological changes of the vesicles detected by TEM. However, it should be taken into account than above LCST the amphiphilic LDBC became entirely hydrophobic and Nile Red tends to keep trapped into the bigger clusters of unstructured material, and it might be not release of the fluorescent probe to the aqueous environment.

Encapsulation in the hydrophilic cavity of the polymeric vesicle and release of Rhodamine B, a fluorescent hydrophilic probe, was also investigated. Vesicles were formed in presence of the cargo fluorescent molecules and light-stimulated release of Rhodamine B was followed by confocal microscopy (Figure 13). Before UV irradiation, many fluorescents spots were visible in the sample due the encapsulation of Rhodamine B (Figure 13a). A region of the sample was selected to follow changes on fluorescence after UV illumination during different times, measuring the average emission intensity of different fluorescent spots, taking approx. 200 pixels per spot. It was observed that fluorescence intensity decreased due to the release of the Rhodamine $\mathrm{B}$ to the aqueous media (Figure 13b). No fluorescence was detected after $5 \mathrm{~min}$. An additional experiment was carried to study the effect of temperature. Vesicles were heated over LCST for $1 \mathrm{~h}$ and no fluorescent dots were observed by confocal microscopy. This should be related again to the release of Rhodamine B into aqueous media, due to the stimulated morphological changes induced in the polymeric assemblies by the temperature. 


\section{Conclusions}

A new LDBC was prepared by coupling a thermoresponsive linear polymer, with a LCST close to physiological temperature, and the $4^{\text {th }}$ generation of a photoresponsive bis-MPA based dendron that was co-functionalized at the periphery with an alkyl chain and 4-isobutyloxyazobenzene in a 50:50 molar ratio. This LDBC was affordable by CuAAC and characterized by ${ }^{1} \mathrm{H}-\mathrm{RMN}$, FTIR and GPC to ensure the adequate coupling of the preformed blocks.

This LDBC self-assembled in water to form vesicles whose morphological changes upon UV-light irradiation or when heated above the LSCT of the thermoresponsive linear block were studied by TEM. Using UV-vis spectroscopy changes upon UV illumination were associated to $E-Z$ photoisomerization of the azobenzene.

The effect of light and temperature was real-time monitored by SAXS and WAXS. SAXS measurements were consistent with the presence of polymeric vesicles having a diameter in accordance with TEM and DLS. By SAXS the effect on the vesicles size upon light stimulation was observed. By WAXS both, the effect of the light and the temperature, on the interchain distances was tracked. Results point to a densification of the polymer chains triggered by UV illumination or by overpassing the LCST, and associated to the morphological changes observed by TEM.

Finally, it was demonstrated the ability of the vesicles to encapsulate both, hydrophobic and hydrophilic molecules and the possibility of controlling their release under light and temperature stimuli.

\section{Acknowledgements}

This work was supported by the MINECO, Spain under the projects MAT2014-55205P, MAT2014-59187-R, MAT2012-33517 and FEDER. Financial support from 
Gobierno de Aragón and Fondo Social Europeo is also acknowledged. The authors would like to acknowledge the Servicios Generales de Apoyo a la Investigación - SAI and the Advanced Microscopy Laboratory - LMA of the Universidad de Zaragoza for the TEM observations. The authors additionally acknowledge the use of the CEQMA Services of the Universidad de Zaragoza-CSIC. H. García-Juan acknowledges Gobierno de Aragón for his $\mathrm{PhD}$ grant. 


\section{REFERENCES}

[1] W. Jiang, Y. Zhou, D. Yan, Chem. Soc. Rev. 2015, 44, 3874.

[2] F. Meng, Z. Zhong, J. Feijen, Biomacromolecules 2009, 10, 197.

[3] R. Cheng, F. Meng, C. Deng, H.-A. Klok, Z. Zhong, Biomaterials 2013, 34, 3647.

[4] D. Bléger, S. Hecht, Angew. Chem. Int. Ed. 2015, 54, 11338.

[5] G. Vancoillie, D. Frank, R. Hoogenboom, Prog. Polym. Sci. 2014, 39, 1074.

[6] J.-F. Lutz, A. Hoth, Macromolecules 2006, 39, 893.

[7] E. Blasco, M. Piñol, L. Oriol, Macromol. Rapid Commun. 2014, 35, 1090.

[8] K. Matyjaszewski, Macromolecules 2012, 45, 4015.

[9] J. del Barrio, L. Oriol, C. Sánchez, J. L. Serrano, A. Di Cicco, P. Keller, M.-H. Li, J. Am. Chem. Soc. 2010, 132, 3762.

[10] E. Blasco, J. del Barrio, C. Sánchez-Somolinos, M. Piñol, L. Oriol, Polym. Chem. 2013, 4, 2246.

[11] E. Blasco, J. L. Serrano, M. Piñol, L. Oriol, Macromolecules 2013, 46, 5951.

[12] F. J. Balta-Calleja, C. G. Vonk, X-ray Scattering of Synthetic Polymers; ELSEVIER: Amsterdam, 1989.

[13] Applications of Synchrotron Light to Scattering and Diffraction in Materials and Life Sciences. Lecture Notes in Physics 776. T. A. Ezquerra, M. C. GarcíaGutiérrez, A. Nogales, M. Gómez Eds., Springer-Verlag: Berlin, 2009.

[14] R. H. Staff, J. Willersinn, A. Musyanovych, K. Landfester, D. Crespy, Polym. Chem. 2014, 5, 4097.

[15] H. G. Schild, D. A. Tirrell, Langmuir 1991, 7, 665.

[16] J. L. Mynar, A. P. Goodwin, J. A. Cohen, Y. Ma, G. R. Fleming, J. M. J. Fréchet, Chem. Commun. 2007, 2081.

[17] A.P. Lopez-Oliva, N. J. Warren, A. Rajkumar, O.O. Mykhaylyk, M. J. Derry, K. E. B. Doncom, M. J. Rymaruk, S. P. Armes, Macromolecules 2015, 48, 3547. 
[18] N. J. Warren, O. O. Mykhaylyk, A. J. Ryan, M. Williams, T. Doussineau, P. Dugourd, R. Antoine, G. Portale, S. P. Armes, J. Am. Chem. Soc. 2015, 137, 1929.

[19] L. A. Feigin, D. I. Svergun, Structure analysis by small angle X-ray and neutron scattering; Penum Press: New York, 1987.

[20] O. Glatter, O. Kratky, Small angle X ray scattering; Academic Press: London, 1982.

[21] G. Beaucage, J. Appl. Crystallography 1995, 28, 717.

[22] R. Hernández, J. Sacristán, A. Nogales, T.A.Ezquerra, C.Mijangos, Langmuir 2009, 25, 13212-13218.

[23] G.R. Mitchell, In Comprehensive Polymer Science, G. Allen, J. C. Bevington, C. Booth, C. Price, Eds.; Pergamon Press: London, 1989; Vol. 1.

[24] J. J. del Val, J. Colmenero, J. Non-Cryst. Sol. 1998, 232-234, 377.

[25] J. J. del Val, J. Colmenero, B. Rosi, G. R. Mitchell, Polymer 1995, 36, 3625. 


\section{FIGURE CAPTIONS}

Figure 1. Chemical structure of (OEG-stat-DEG)_3500-block-d(isoAZO/C18)

Figure 2. ${ }^{1} \mathrm{H}$ RMN spectrum $\left(400 \mathrm{MHz}, \mathrm{CDCl}_{3}\right)$ of (OEG-stat-DEG)_3500. Values of $\mathrm{x}$ and $\mathrm{y}$ were calculated from peaks labelled $\mathrm{b}$ and $\mathrm{c}$, by using the following system of equations: $I_{b}=3 x+3 y=$ and $I_{c}=32 x+6 y$

Figure 3. ${ }^{1} \mathrm{H}$ NMR spectrum $\left(400 \mathrm{MHz}, \mathrm{CDCl}_{3}\right)$ of (a) d(isoAZO/C18), (b)(OEG-statDEG)_3500 and (c) (OEG-stat-DEG)_3500-block-d(isoAZO/C18). Amplification of terminal group peaks are represented in the insets of (a) and (b) corresponding to the preformed blocks. Initial peaks are not observed after coupling, see insets in (c).

Figure 4. TEM images of (OEG-stat-DEG)_3500-block-d(isoAZO/C18) selfassemblies: (a) original self-assemblies, (b) after UV illumination for $10 \mathrm{~min}$, (c) heated above LCST and (d) heated above LCST and slowly cooled down to room temperature.

Figure 5. UV-vis spectra of the vesicles suspension before and after irradiation with UV light for different times

Figure 6. SAXS intensity, after substraction of water scattering, as a function of the scattering vector. $(\bullet)$ Before UV illumination, and $(O)$ after 10 min of UV illumination. Inset, example of the Guinier plot to estimate the vesicle diameter.

Figure 7. Dependence of the estimated vesicle diameter $D$ as a function of the UV illumination time. Continuous line is a guide to the eyes.

Figure 8. WAXS intensity as a function of the scattering vector, after water subtraction, before UV illumination (black), and after 10 min of UV illumination (red).

Figure 9. Evolution of the interchain average distance $d_{\text {inter }}$, obtained from the first maximum of the WAXS profile, as a function of UV illumination time.

Figure 10. WAXS profile of the vesicles suspension including water contribution during heating (up) and cooling scan (down). 
Figure 11. Dependence with temperature of (a) the average interchain distance, $d_{\text {inter, }}$, and (b) the intensity of the main peak in WAXS ( $q$ around $19 \mathrm{~nm}^{-1}$ ), upon heating ( $\left.\square\right)$, and upon cooling $(\bullet)$.

Figure 12. Emission spectra evolution of Nile Red loaded vesicle upon illumination (a) and on heating (b)

Figure 13. (a) Confocal image of Rhodamine B loaded vesicles, (b) average fluorescence intensity of selected spots at different irradiation times. 


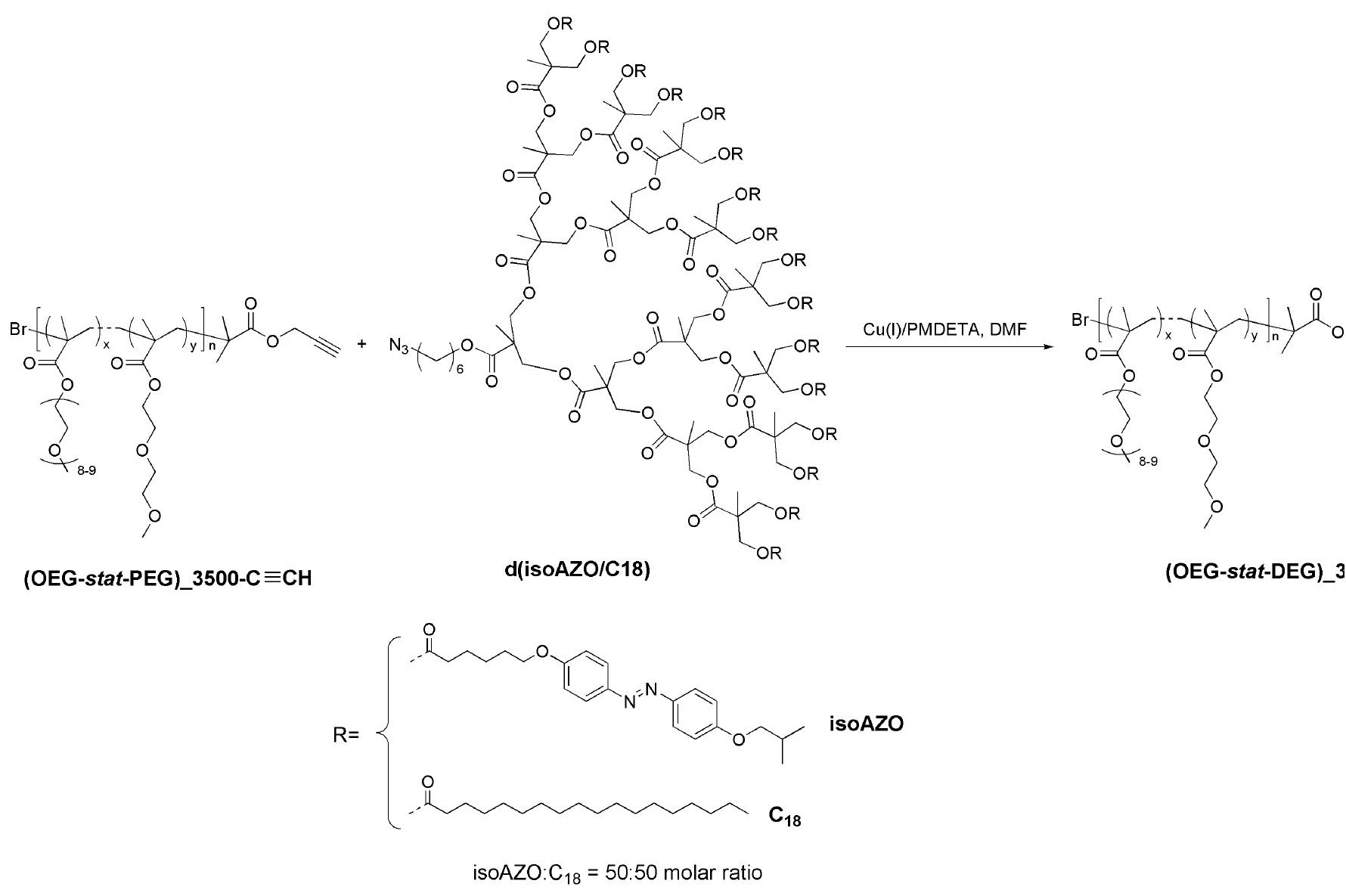

Figure 1. Chemical structure of (OEG-stat-DEG)_3500-block-d(isoAZO/C18) 


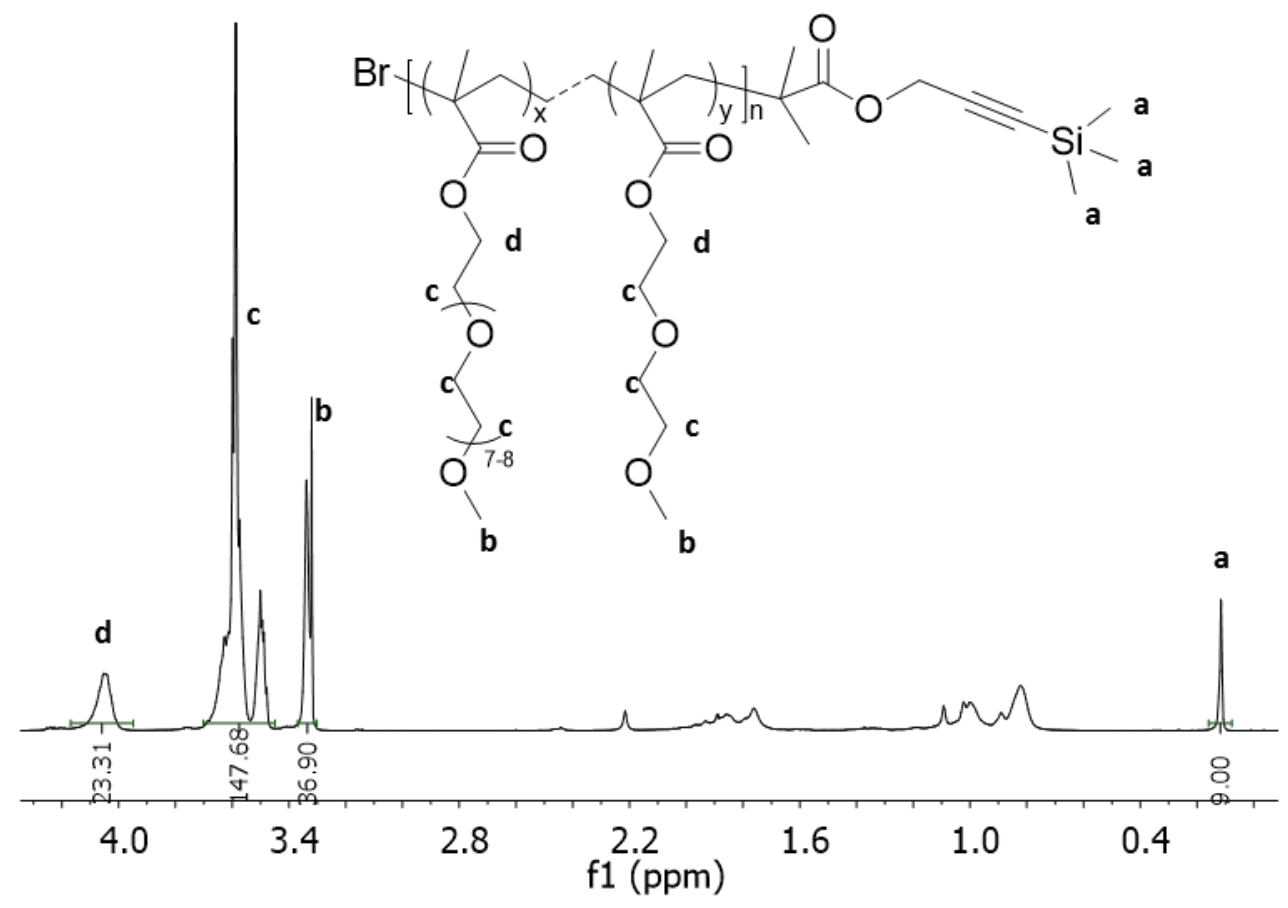

Figure 2. ${ }^{1} \mathrm{H}$ RMN spectrum (400 MHz, $\left.\mathrm{CDCl}_{3}\right)$ of (OEG-stat-DEG)_3500. Values of $\mathrm{x}$ and $\mathrm{y}$ were calculated from peaks labelled $\mathrm{b}$ and $\mathrm{c}$, by using the following system of equations: $I_{b}=3 x+3 y=$ and $I_{c}=32 x+6 y$ 
(a)

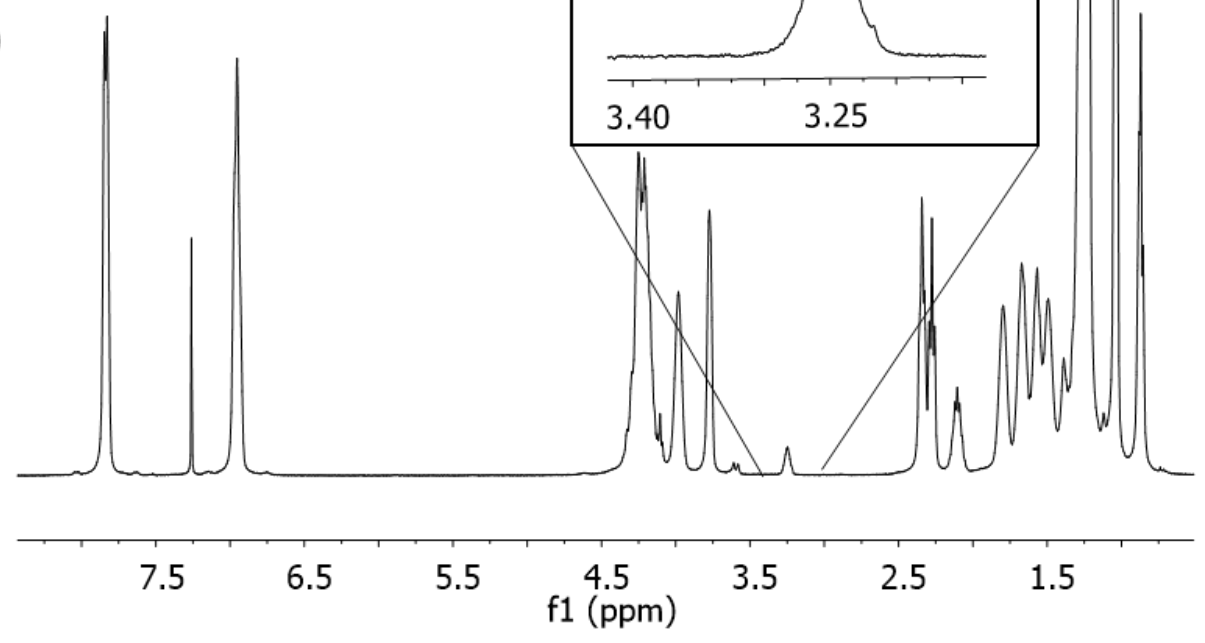

(b)

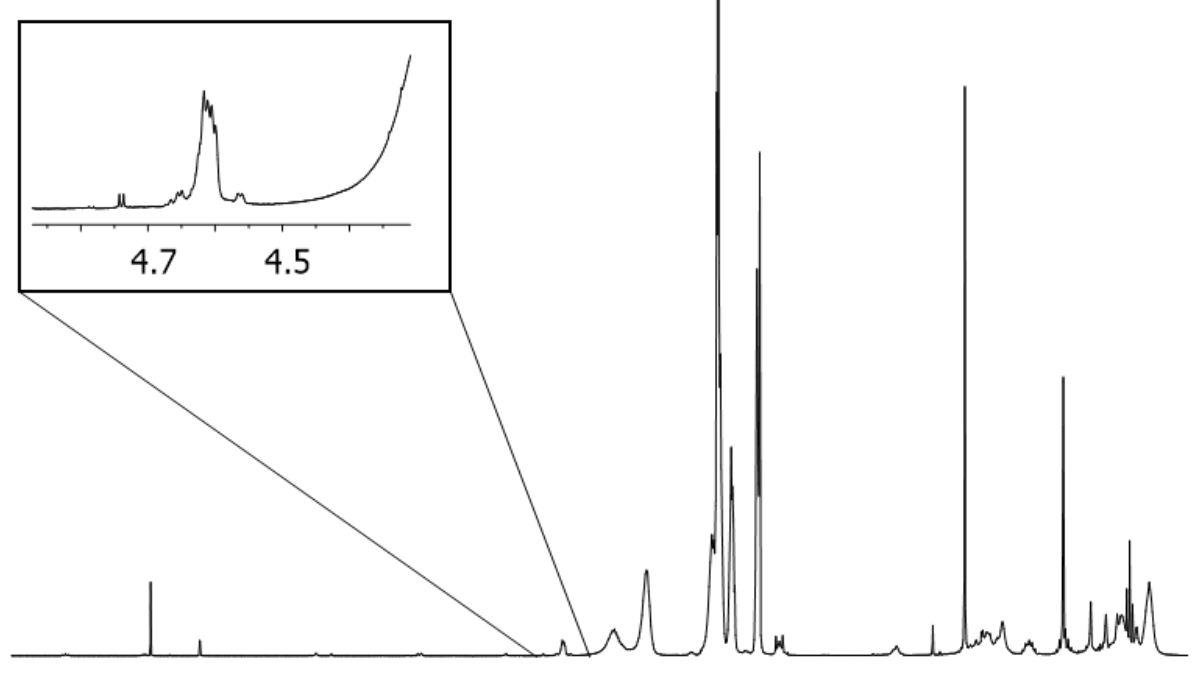

(c)

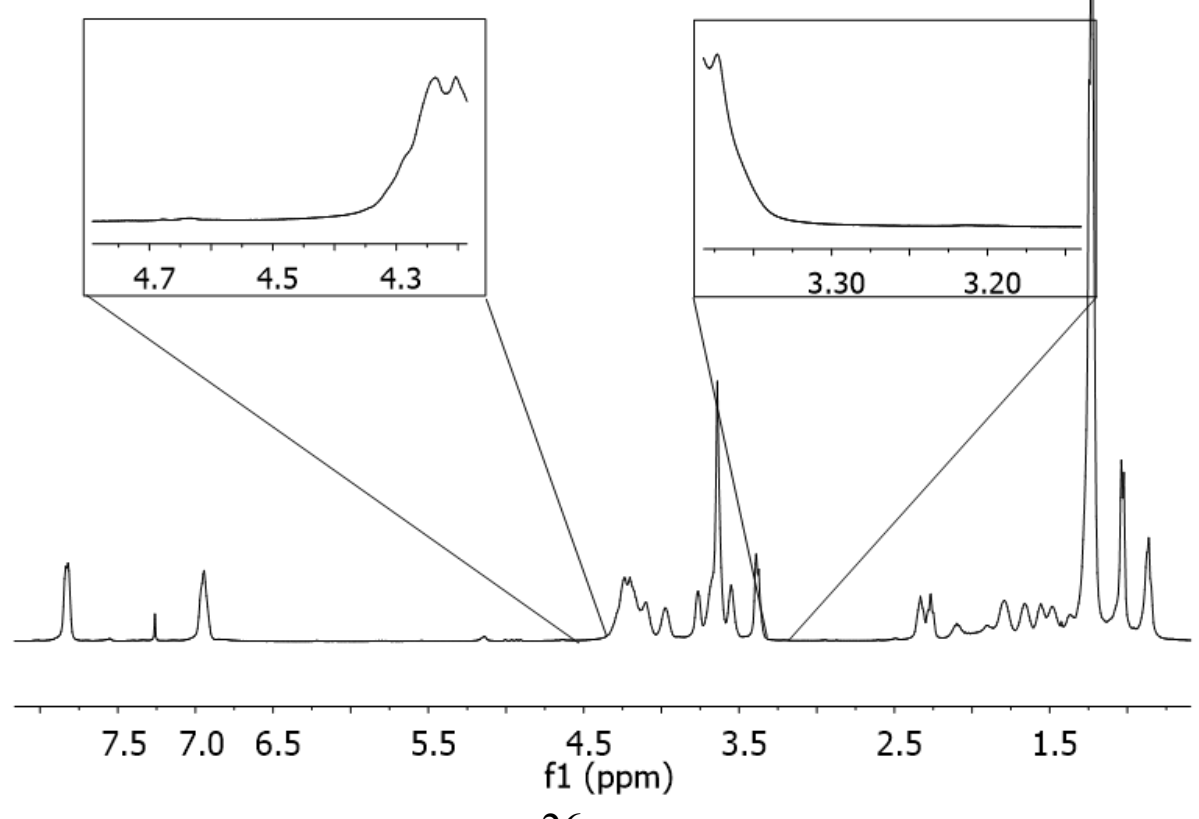

- 26 - 
Figure 3. ${ }^{1} \mathrm{H}$ NMR spectrum $\left(400 \mathrm{MHz}, \mathrm{CDCl}_{3}\right)$ of (a) d(isoAZO/C18), (b)(OEG-statDEG)_3500 and (c) (OEG-stat-DEG)_3500-block-d(isoAZO/C18). Amplification of terminal group peaks are represented in the insets of (a) and (b) corresponding to the preformed blocks. Initial peaks are not observed after coupling, see insets in (c). 
(a)

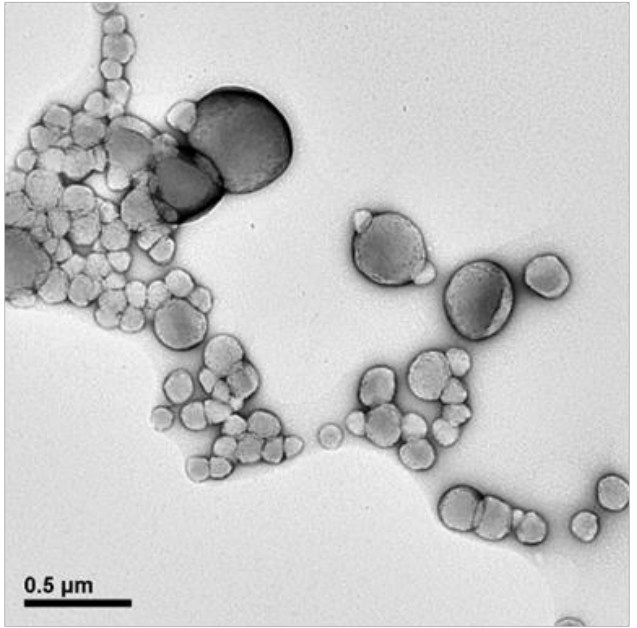

(c)

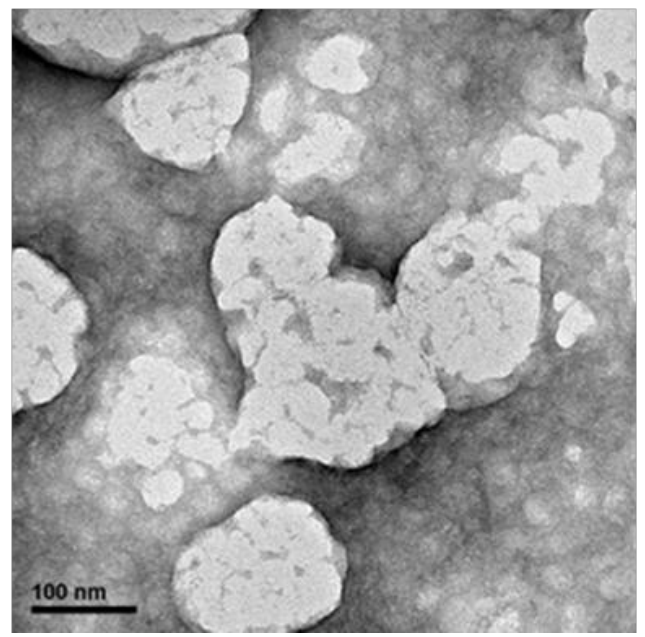

(b)

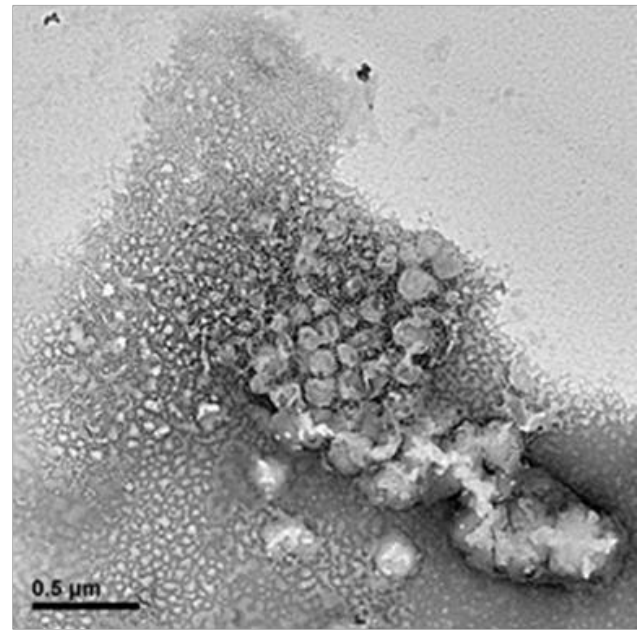

(d)

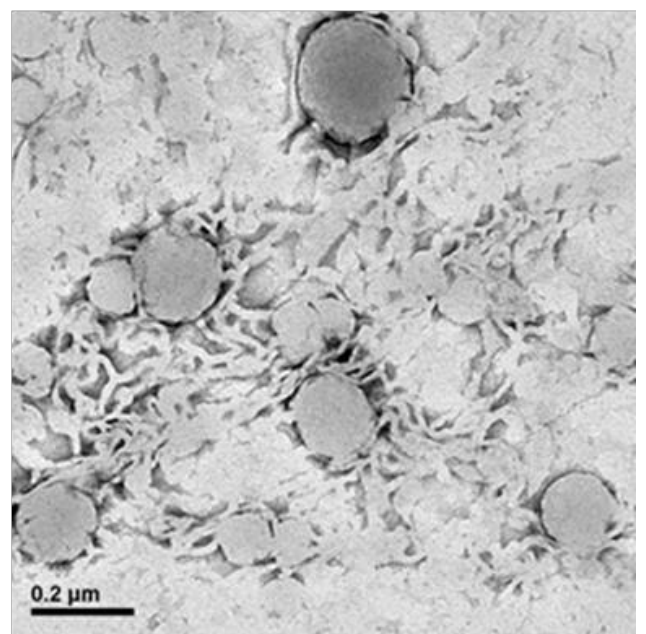

Figure 4. TEM images of (OEG-stat-DEG)_3500-block-d(isoAZO/C18) selfassemblies: (a) original self-assemblies, (b) after UV illumination for $10 \mathrm{~min}$, (c) heated above LCST and (d) heated above LCST and slowly cooled down to room temperature. 


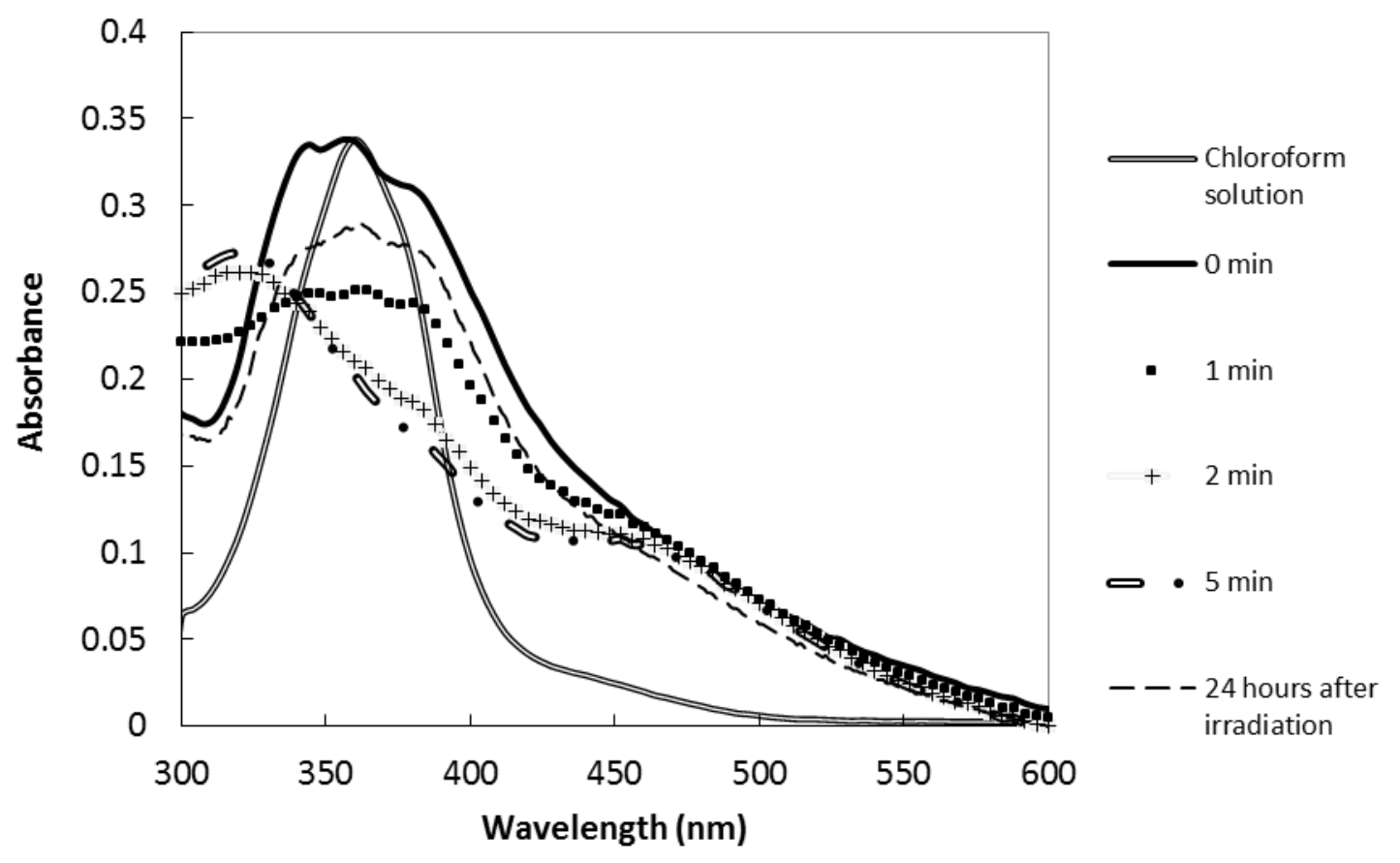

Figure 5. UV-vis spectra of the vesicles suspension before and after irradiation with UV light for different times 


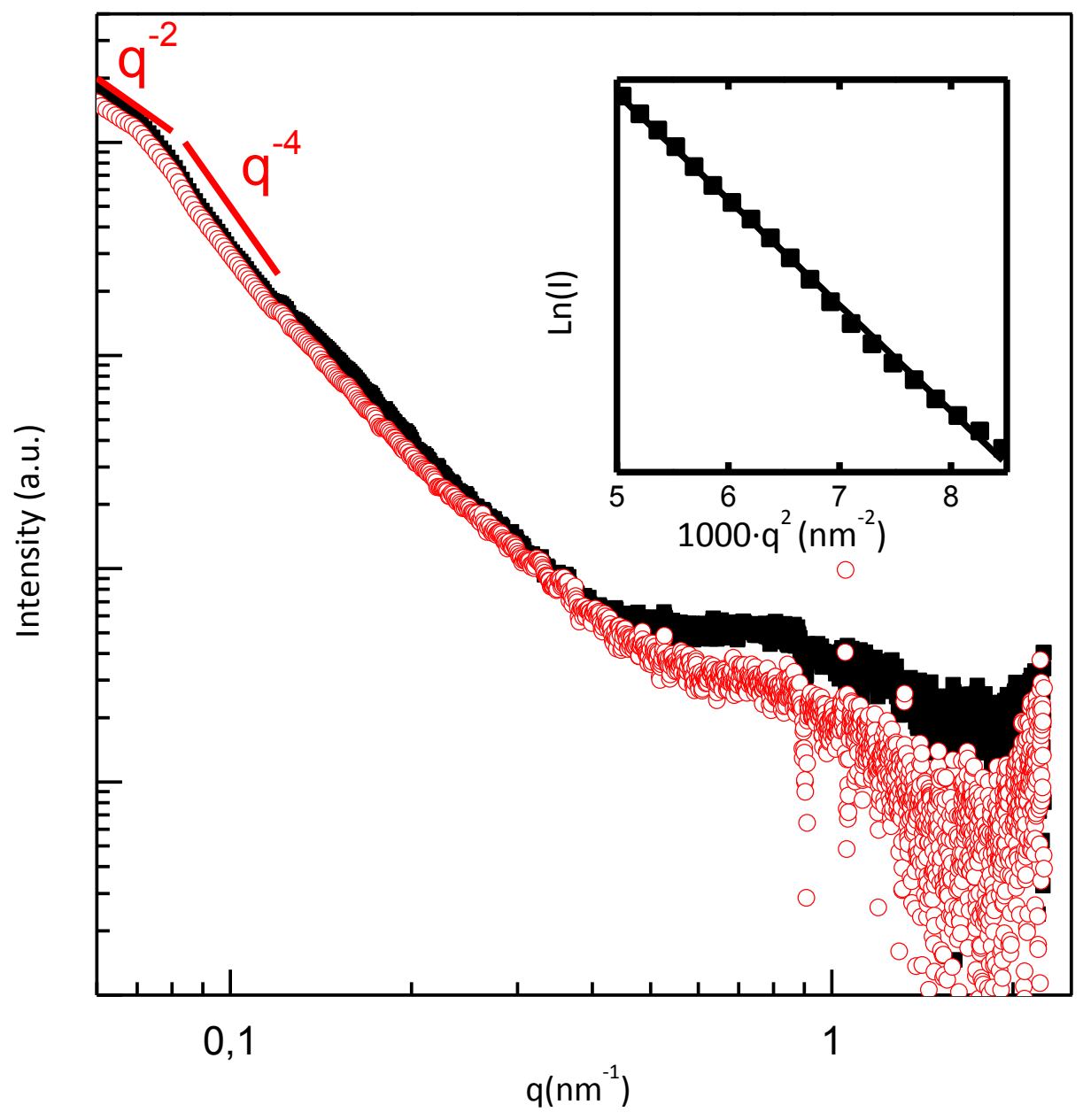

Figure 6. SAXS intensity, after substraction of water scattering, as a function of the scattering vector. $(\bullet)$ Before UV illumination, and $(\mathrm{O})$ after 10 min of UV illumination. Inset, example of the Guinier plot to estimate the vesicle diameter. 


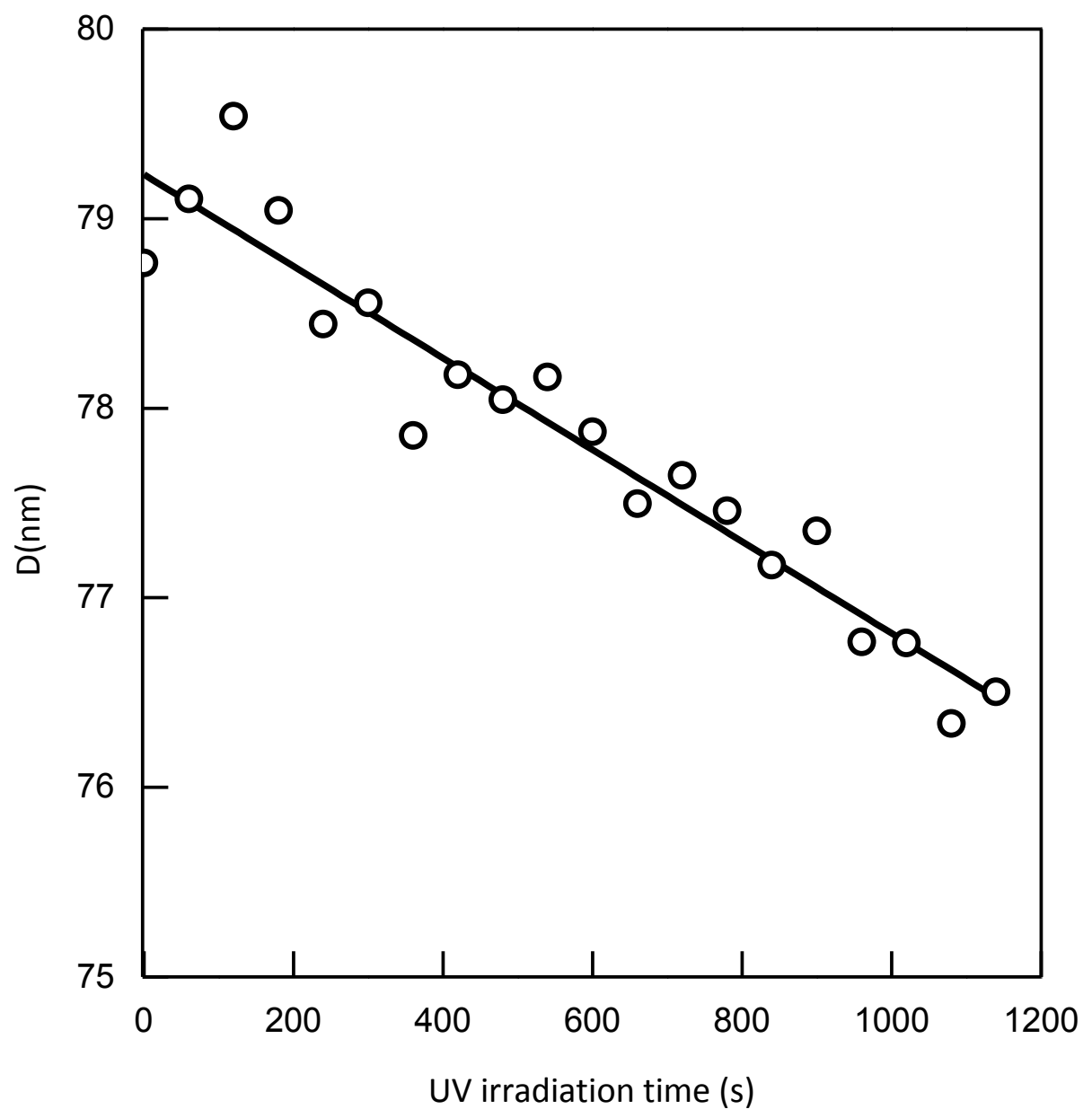

Figure 7. Dependence of the estimated vesicle diameter $D$ as a function of the UV illumination time. Continuous line is a guide to the eyes. 


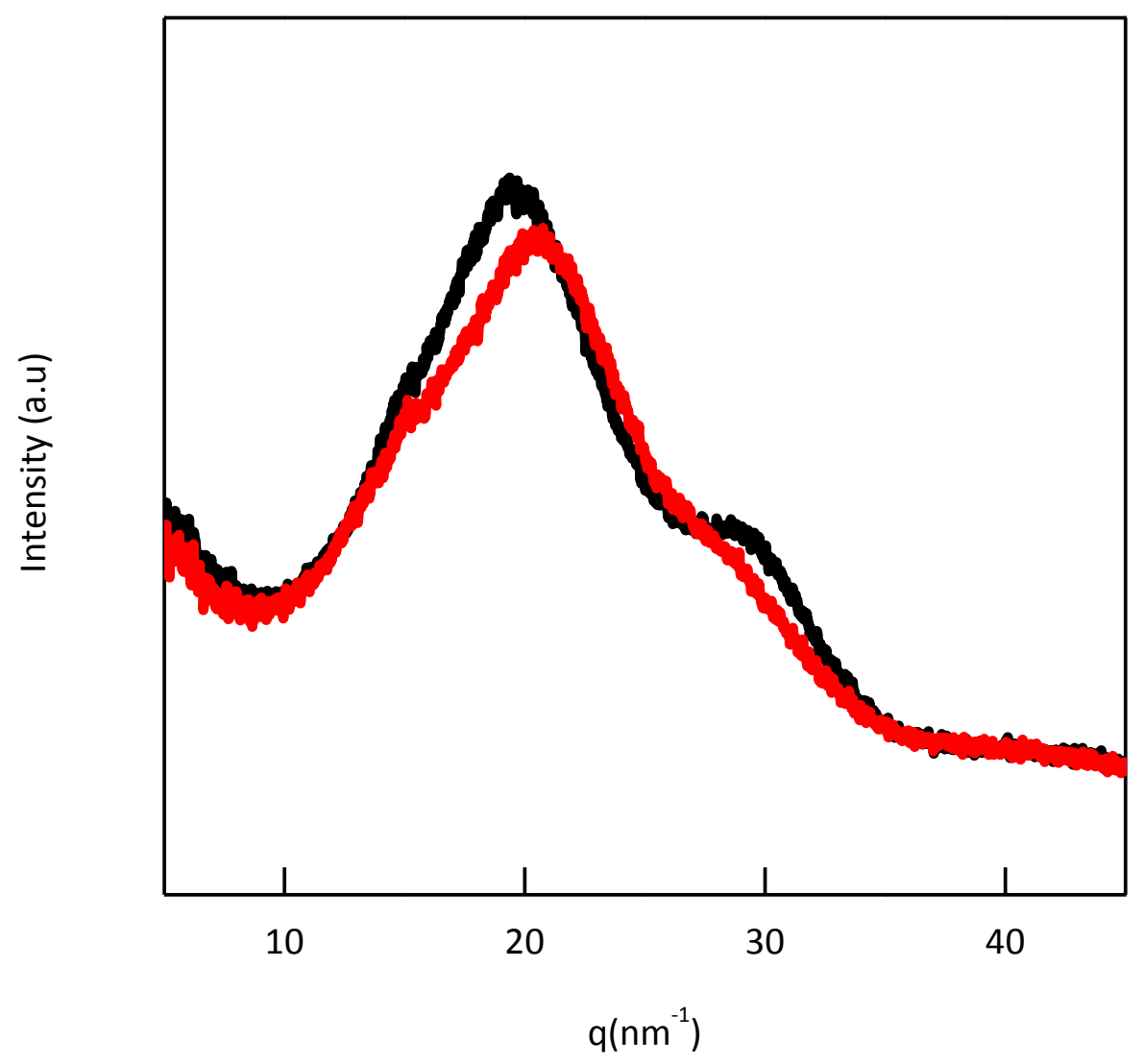

Figure 8. WAXS intensity as a function of the scattering vector, after water subtraction, before UV illumination (black), and after 10 min of UV illumination (red). 


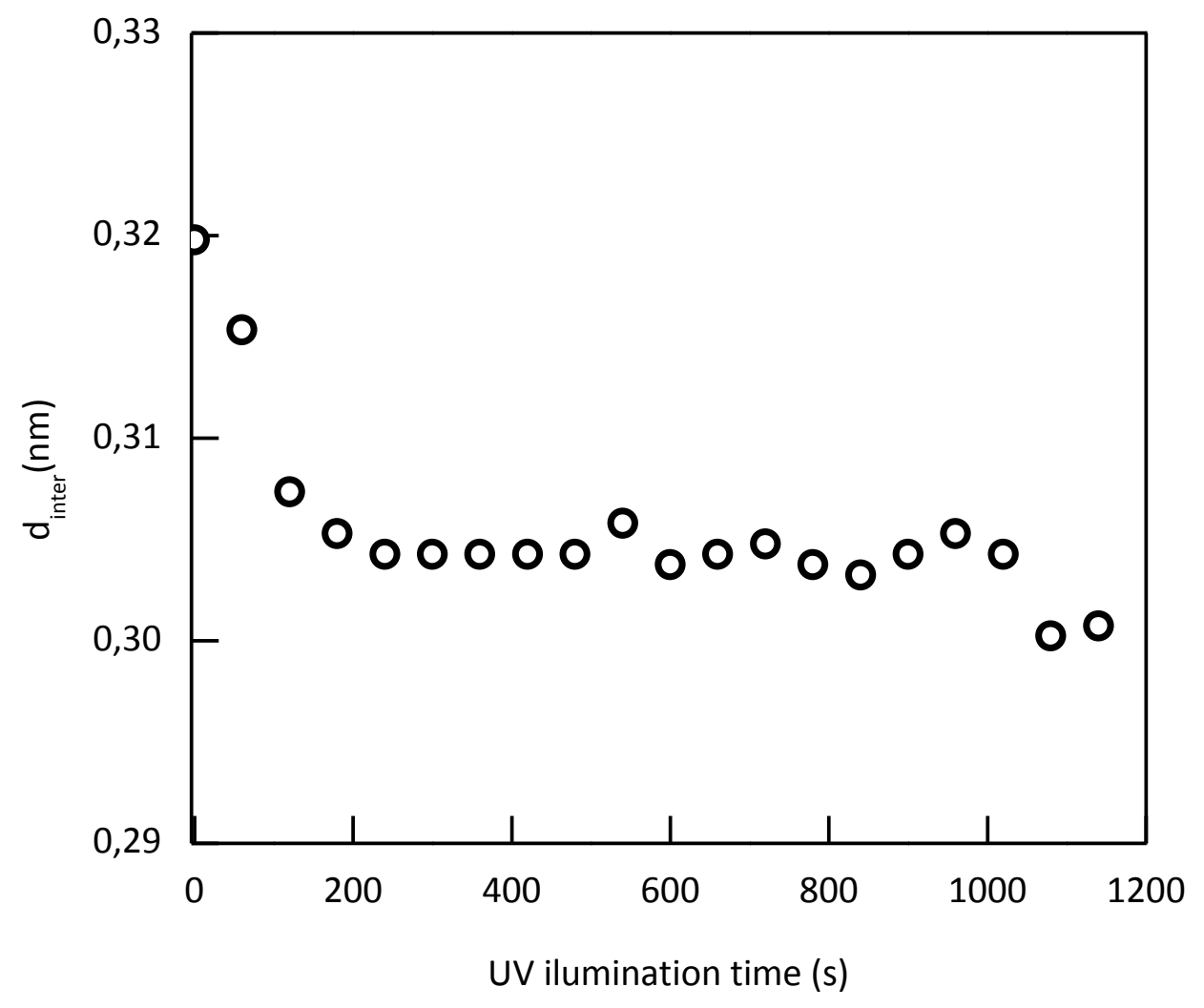

Figure 9. Evolution of the interchain average distance $d_{\text {inter }}$, obtained from the first maximum of the WAXS profile, as a function of UV illumination time. 

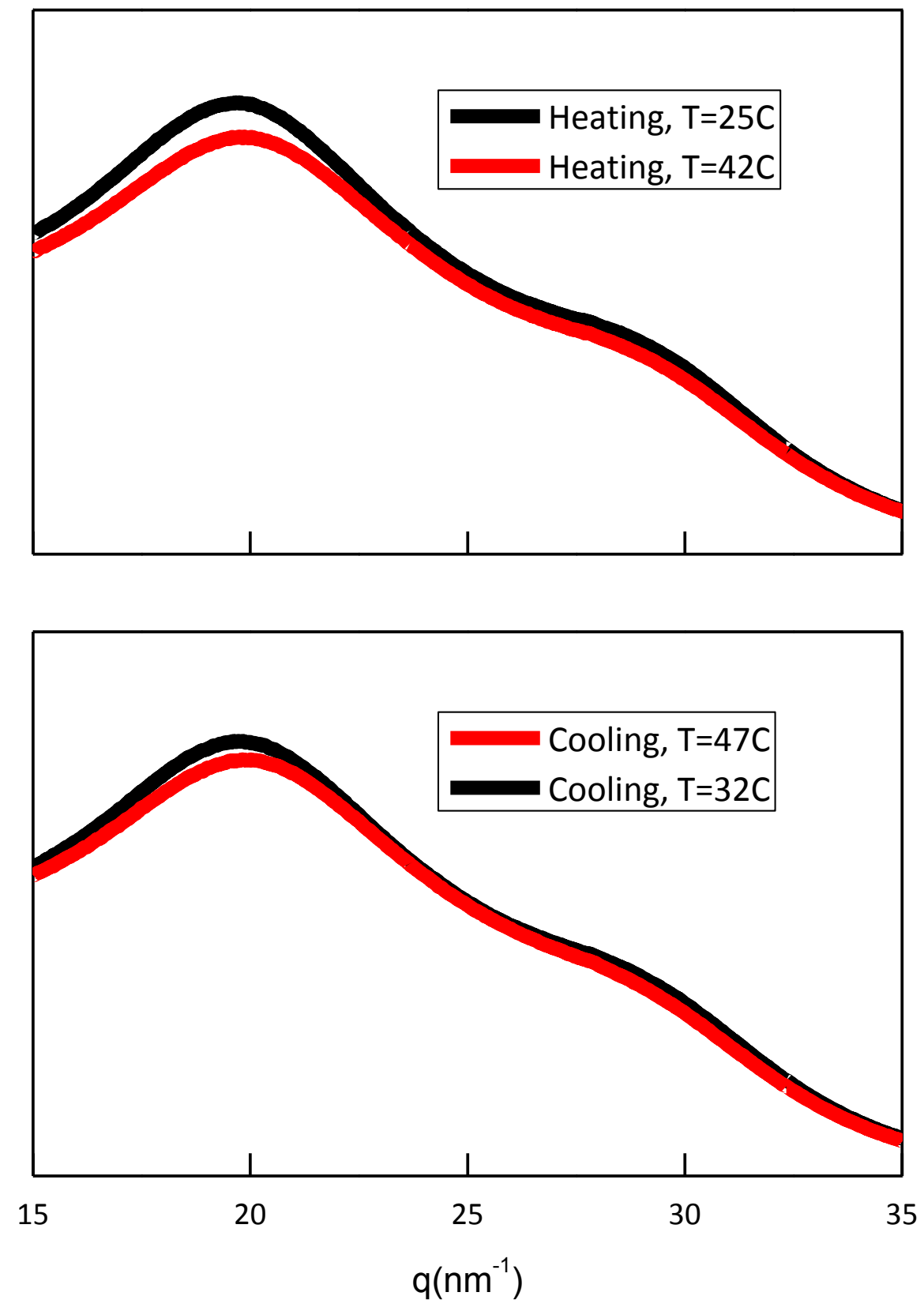

Figure 10. WAXS profile of the vesicles suspension including water contribution during heating (up) and cooling scan (down). 

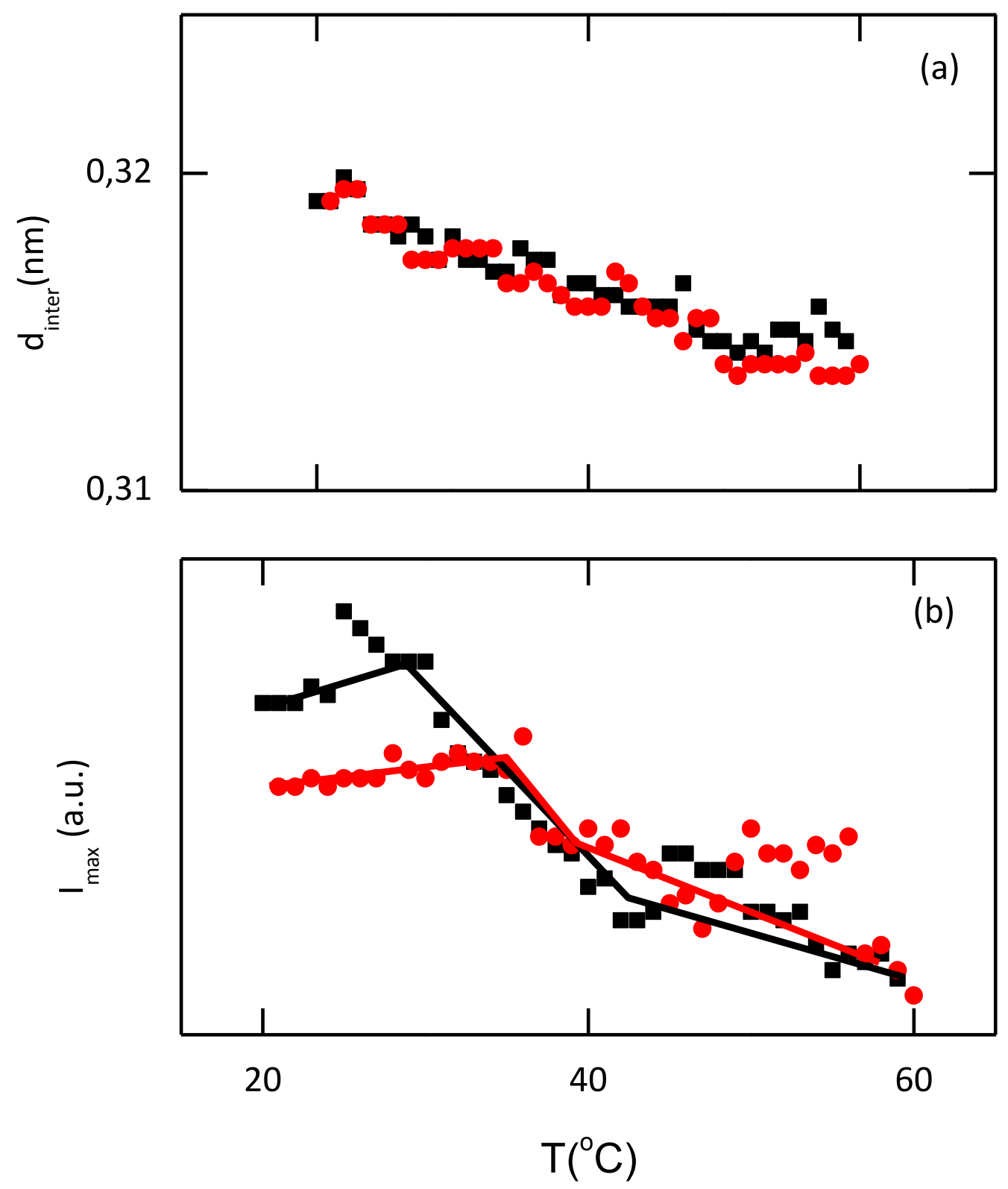

Figure 11. Dependence with temperature of (a) the average interchain distance, $d_{\text {inter, }}$, and (b) the intensity of the main peak in WAXS ( $q$ around $\left.19 \mathrm{~nm}^{-1}\right)$, upon heating ( $\left.\mathbf{\square}\right)$, and upon cooling $(\bullet)$. 

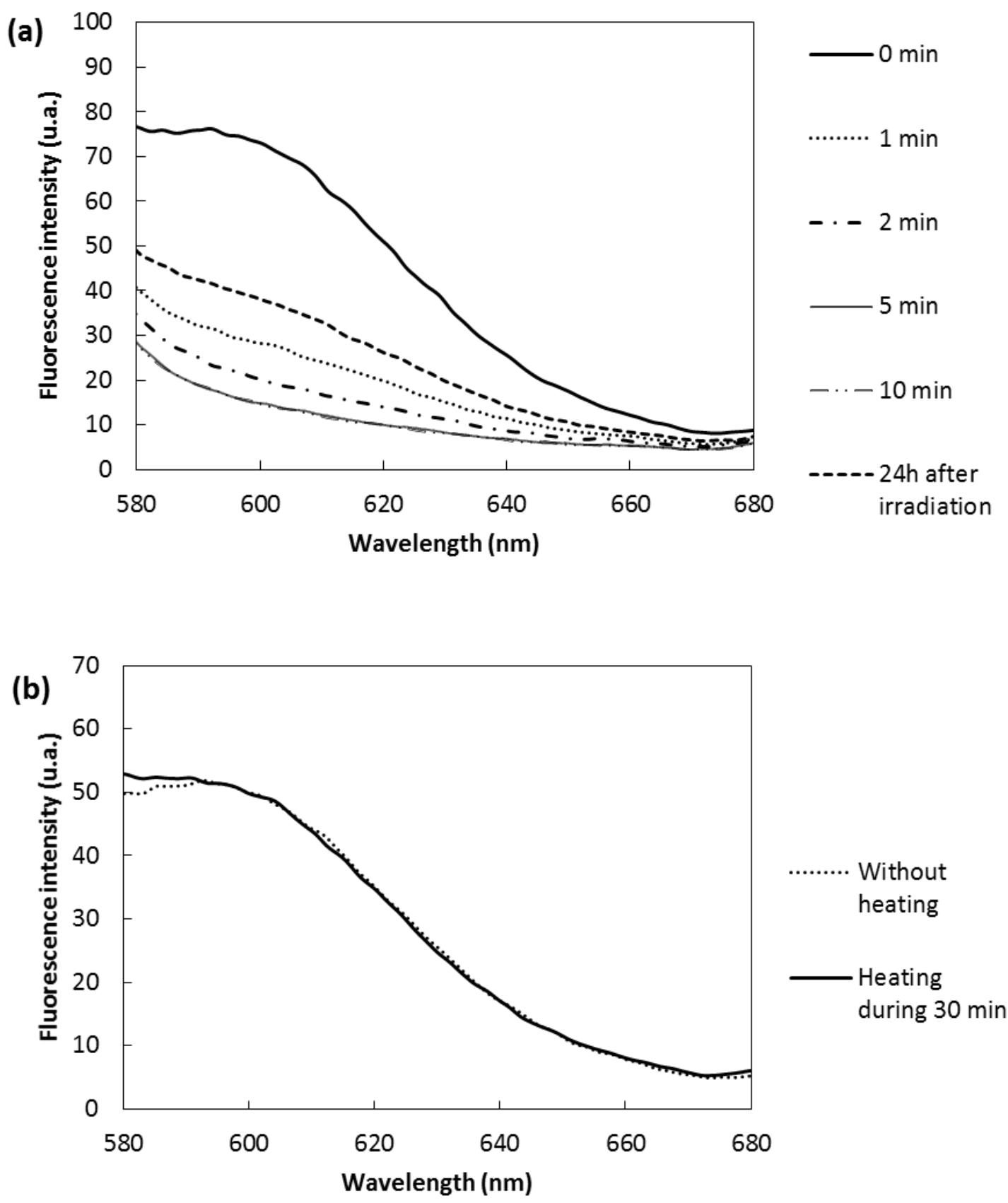

Figure 12. Emission spectra evolution of Nile Red loaded vesicle upon illumination (a) and on heating (b) 
(a)
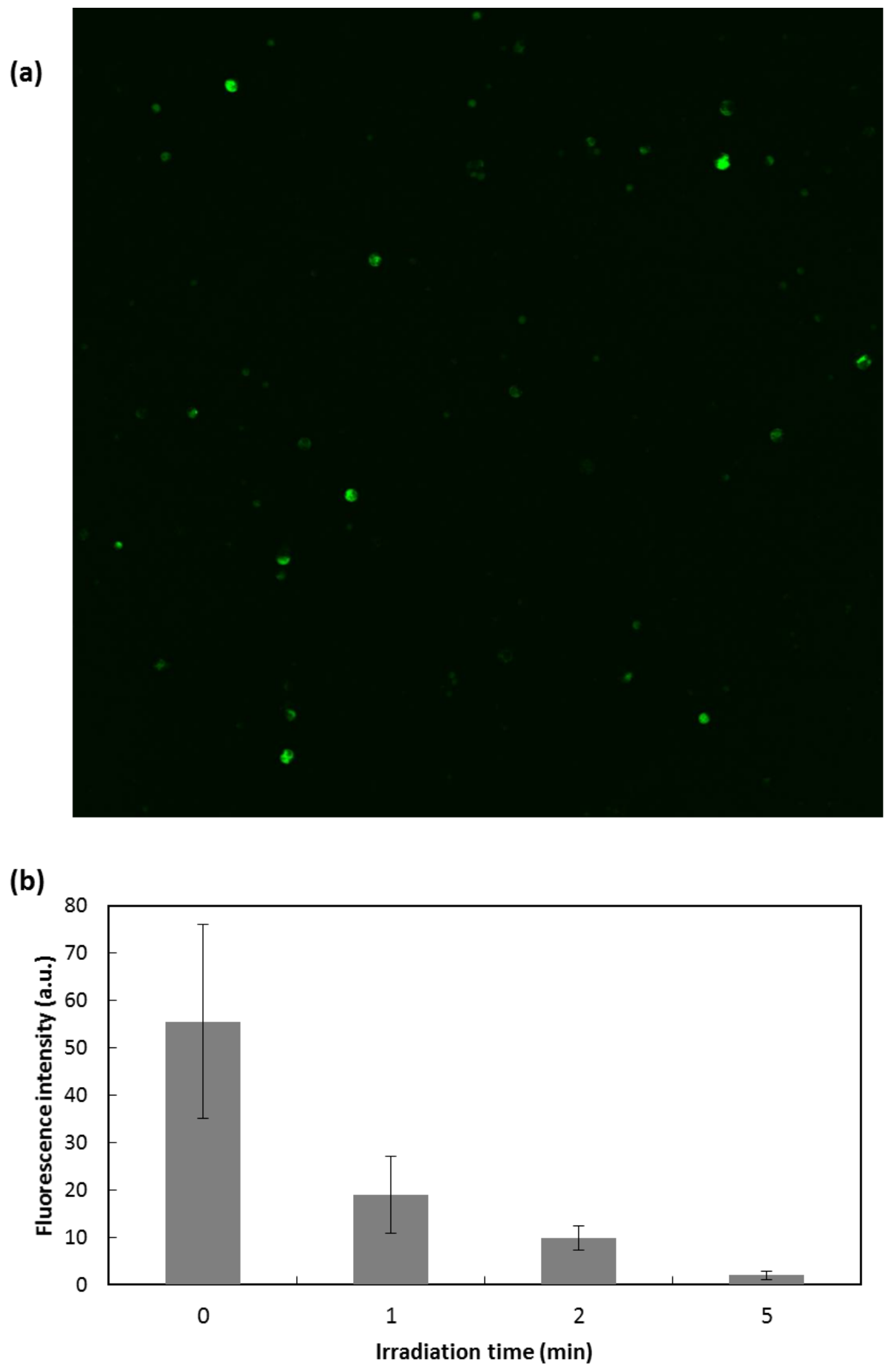

Figure 13. (a) Confocal image of Rhodamine B loaded vesicles, (b) average fluorescence intensity of selected spots at different irradiation times. 